\title{
COFINAL FAMILIES OF BOREL EQUIVALENCE RELATIONS AND QUASIORDERS
}

\author{
CHRISTIAN ROSENDAL
}

\begin{abstract}
Families of Borel equivalence relations and quasiorders that are cofinal with respect to the Borel reducibility ordering, $\leq_{B}$, are constructed. There is an analytic ideal on $\omega$ generating a complete analytic equivalence relation and any Borel equivalence relation reduces to one generated by a Borel ideal. Several Borel equivalence relations, among them Lipschitz isomorphism of compact metric spaces, are shown to be $K_{\sigma}$ complete.
\end{abstract}

$\S 1$. Introduction. For $R$ and $S$ binary relations on Polish spaces $X$ and $Y$, respectively, one writes $R \leq_{B} S$ and says that $R$ Borel reduces to $S$, if there is a Borel function $f: X \rightarrow Y$ such that $x R y \Longleftrightarrow f(x) S f(y)\left(\right.$ iff $\left.(f \times f)^{-1}(S)=R\right)$. Usually this ordering has been studied when $R$ and $S$ both are equivalence relations, but recently it has turned out that the study of quasiorders, i.e., reflexive, transitive relations, is important for the structure theory of $\leq_{B}$ on analytic equivalence relations. For $R$ a quasiorder on $X$ one denotes by $\equiv_{R}$ the corresponding equivalence relation, $x \equiv_{R} y \Longleftrightarrow x R y \wedge y R x$. Then $R \leq_{B} S$ can be seen as saying that there is a function, admitting a Borel lifting, from $X / \equiv_{R}$ into $Y / \equiv_{S}$ that embeds the partial ordering induced by $R$ into the partial ordering induced by $S$. This means that the objects in $X$ are simpler to classify with respect to $R$ than the objects in $Y$ with respect to $S$. It becomes more explicit when both $R$ and $S$ are equivalence relations, where $R \leq_{B} S$ implies that $Y$ objects modulo $S$ provide complete invariants for $X$ objects with respect to $R$-equivalence. And furthermore, the invariants can be calculated in a Borel manner from the initial objects.

A classical example of this is the Stone representation of (countable) Boolean algebras by compact (Polish) spaces up to homeomorphism. This shows that the isomorphism relation of countable Boolean algebras Borel reduces to homeomorphism of compact Polish spaces.

In the article [16], by Louveau and the author, it was shown that certain naturally occurring quasiorders such as embeddability between binary relations on a countable set are analytic complete, i.e., Borel reduce any other analytic quasiorder. We use these results to show that certain families of Borel quasiorders and equivalence relations are cofinal with respect to $\leq_{B}$.

Families of Borel equivalence relations, cofinal for the the ones reducing to isomorphism of countable structures (that is, classified by countable structures), were already given by Friedman and Stanley in [5]. Their main example is the

Received November 14, 2003; revised June 24, 2005. 
class consisting of the relation of isomorphism between trees of bounded height less than $\xi$, with $\xi$ running over the countable ordinals. But, as has been known for long, these families fail to reduce even quite simple Borel equivalence relations. A particular example of such an equivalence relation is $E_{1}$ defined on $\mathbb{R}^{\omega}$ by

$$
x E_{1} y \Longleftrightarrow \exists N \forall n \geq N x_{n}=y_{n} .
$$

Our results are a happy generalisation of the analysis in [5] combined with the completeness theorem in [16].

Given an ideal $I$ on $\omega$, there is a naturally associated equivalence relation $E_{I}$ on $2^{\omega}$ defined by $x E_{I} y \Longleftrightarrow x \Delta y \in I$. We construct an analytic ideal $I_{\max }$ generating a complete analytic equivalence relation. This, combined with a rank argument, shows that any Borel equivalence relation reduces to one generated by a Borel ideal.

Another class of equivalence relations that admits complete elements is that of $K_{\sigma}$ 's. One example was given by Kechris in [10] and many more by Louveau and the author in [16]. These all had the disadvantage that they were presented in the form $\equiv_{R}$, for some complete $K_{\sigma}$ quasiorder $R$. This in turn implies that they are very difficult to reduce to anything else, unless this itself is given as the equivalence relation associated to some quasiorder. The same is the case with complete analytic equivalence relations, where it can even be shown that $E$ is complete iff $E=\equiv_{R}$ for some complete quasiorder $R$. So the only naturally occurring equivalence relations known to be $K_{\sigma}$ complete were of the form: biembeddability of finitely branching combinatorial trees or isometric biembeddability of certain classes of Polish metric spaces. We remedy this by giving more handy versions of this important node of the Borel reducibility ordering and subsequently apply our results to classification problems in analysis. In particular, we show that Lipschitz isomorphism of compact metric spaces is $K_{\sigma}$ complete.

The paper is organised as follows. In section 2 we give the construction of the analytic ideal generating a complete analytic equivalence relation. Section 3 concerns operations on equivalence relations and quasiorders, some of which are shown to lead to jumps in the Borel hierarchy. Generalising in section 4 the Scott analysis of isomorphism to quasiorders, we show that a certain jump induces cofinal families among quasiorders and equivalence relations. Finally, section 5 addresses the problem of finding complete $K_{\sigma}$ equivalence relations.

Our general reference for descriptive set theory will be the book of Kechris [11] whose notation will be adopted wholesale and which explains most of the common notions used here, notably concerning trees, Borel sets, etc.

This paper was written while the author was a doctoral student at the University of Paris 6. I am sincerely grateful to Alain Louveau, without whom this would not have been. I also thank Olivier Guédon for showing me the proof of Lemma 27 and the referee for a careful reading of the paper.

§2. A complete analytic ideal. We will show that there is an analytic ideal on $\omega$ such that its induced equivalence relation on $2^{\omega}$ is complete analytic. This will by a reflection argument imply that any Borel equivalence relation reduces to one induced by a Borel ideal and thereby solve a problem of Kanovei. In fact, for our purpose it will be easier to define the ideal on a countable set other than $\omega$, namely $D$, the complete tree on $2 \times \omega$. 
On $\omega^{<\omega}$ we define the following addition and ordering: for $s, t \in \omega^{<\omega}$ of the same length let $(s+t)(k)=s(k)+t(k)$. And for $s, t \in \omega^{<\omega}$ put

$$
s \leq t \Leftrightarrow|s|=|t| \wedge \forall k<|s| s(k) \leq t(k) .
$$

We recall from [15] that a tree $T$ on $2 \times \omega$ is called normal if for any $u \in 2^{n}$ and $s, t \in \omega^{n}$ such that $s \leq t$, we have $(u, s) \in T \Longrightarrow(u, t) \in T$.

The following relations defined on the class $\mathscr{T}$ of normal trees were shown to be respectively a complete analytic quasiorder and a complete analytic equivalence relation in $[15,16]$ :

$$
\begin{array}{r}
T \leq_{\max }^{*} S \Longleftrightarrow \exists \gamma \in \omega^{\omega} \forall n \forall u \in 2^{n} \forall s \in \omega^{n}[(u, s) \in T \rightarrow(u, s+\gamma\lceil n) \in S] \\
T E_{\text {max }}^{*} S \Longleftrightarrow \exists \gamma \in \omega^{\omega} \forall n \forall u \in 2^{n} \forall s \in \omega^{n}[(u, s) \in T \rightarrow(u, s+\gamma\lceil n) \in S] \\
\wedge[(u, s) \in S \rightarrow(u, s+\gamma\lceil n) \in T]
\end{array}
$$

Let $I_{\max }$ be the analytic ideal on $D$ generated by $g\left[E_{\max }^{*}\right]$, where $g(T, S):=T \triangle S$.

We claim that the identity mapping is a reduction of $E_{\max }^{*}$ to $E_{I_{\max }}$, i.e., where $x, y \in 2^{D}$ are $E_{I_{\max }}$ equivalent iff their symmetric difference is in $I_{\max }$. $I_{\max }$.

Proof of claim: By the definition of the ideal it is clear that $T E_{\max }^{*} S \Longrightarrow T \triangle S \in$

Notice that due to the normality of the trees, if $(u, s) \in T \Delta S,(u, t) \notin T \Delta S$ and $s \leq t \leq v$, then $(u, v) \notin T \Delta S$.

Suppose that $T \triangle S \in I_{\max }$. Then there are $T_{1}, \ldots, T_{k}, S_{1}, \ldots, S_{k} \in \mathscr{T}$ such that $T \triangle S \subseteq \bigcup_{i=1}^{k} T_{i} \triangle S_{i}$ and $T_{i} E_{\max }^{*} S_{i}$ as witnessed by $\gamma_{i}$.

Let $n$ be given. We will show that if $(u, s) \in T \Delta S, u \in 2^{n}, s \in \omega^{n}$, then $\left(u, s+\sum_{i=1}^{k} \gamma_{i}\lceil n) \notin T \Delta S\right.$, which is enough to show that $T E_{\max }^{*} S$, as witnessed by $\sum_{i=1}^{k} \gamma_{i}$. In fact, it suffices to show that if $(u, s) \in T \Delta S$, then $(u, s+t) \notin T \Delta S$ for some $t \leq \sum_{i=1}^{k} \gamma_{i}\lceil n$. So suppose not. Since $(u, s) \in T \Delta S$, there is some $i_{1} \in\{1, \ldots, k\}$ such that $(u, s) \in T_{i_{1}} \Delta S_{i_{1}}$ and therefore $\left(u, s+\gamma_{i_{1}} \backslash n\right) \notin$ $T_{i_{1}} \Delta S_{i_{1}}$. As $\left(u, s+\gamma_{i_{1}} \backslash n\right) \in T \Delta S$, there is some $i_{2} \in\{1, \ldots, k\} \backslash\left\{i_{1}\right\}$ such that $\left(u, s+\gamma_{i_{1}}\lceil n) \in T_{i_{2}} \Delta S_{i_{2}}\right.$ and again $\left(u, s+\gamma_{i_{1}}\left\lceil n+\gamma_{i_{2}}\lceil n) \notin T_{i_{2}} \Delta S_{i_{2}}\right.\right.$. This continues until we eventually find that $\left(u, s+\sum_{j=1}^{k} \gamma_{i_{j}}\lceil n)=\left(u, s+\sum_{i=1}^{k} \gamma_{i}\lceil n) \in\right.\right.$ $T \triangle S$. But $\left(u, s+\sum_{i=1}^{k} \gamma_{i}\lceil n) \notin \bigcup_{i=1}^{k} T_{i} \Delta S_{i}\right.$ which is a contradiction.

We have proved:

THEOREM 1. $E_{I_{\max }}$ is a complete analytic equivalence relation.

Suppose that $I$ is an analytic ideal on $\omega$ and $C \subseteq 2^{\omega}$ an analytic set disjoint from $I$ and define the following property on $\mathscr{P}\left(2^{\omega}\right) \times \mathscr{P}\left(2^{\omega}\right)$ :

$$
\begin{aligned}
\Phi(A, B) \Longleftrightarrow & A \cap C=\emptyset \wedge \forall x \subseteq y \subseteq \omega(y \in A \rightarrow x \notin B) \wedge \\
& \forall x, y \subseteq \omega(x \in A \wedge y \in A \rightarrow x \cup y \notin B)
\end{aligned}
$$

Then $\Phi$ is $\Pi_{1}^{1}$ on $\Sigma_{1}^{1}$, hereditary and continuous upwards in the second variable. Moreover, $\Phi(I, C I)$, so by the second reflection theorem (see [11, (35.16)]) there is some Borel set $J \supseteq I$ satisfying $\Phi(J, C J)$. $J$ is therefore a Borel ideal containing $I$ and disjoint from $C$. This shows that if $I=\bigcap_{\omega_{1}} B_{\xi}$ are the complements of the Borel sets constituting $\complement I$, then for a closed unbounded set of $\xi<\omega_{1}, B_{\xi}$ is a Borel ideal.

THEOREM 2. Any Borel equivalence relation reduces to one induced by a Borel ideal. 
Proof. Suppose $E$ is a Borel equivalence relation on a Polish space $X$ and $f: X \rightarrow 2^{D}$ is a Borel reduction of $E$ to $E_{I_{\max }}$. Let $I_{\xi}, \xi<\omega_{1}$, be an increasing enumeration of those of the complements of the constituents of $I_{\max }$ that are Borel ideals, and notice that if $x E y$, then $f(x) \Delta f(y) \in I_{\max } \subseteq I_{\xi}$. On the other hand, $\{f(x) \Delta f(y) \mid \neg x E y\}$ is an analytic set disjoint from $I_{\max }$ and hence also disjoint from some $I_{\xi}$. Therefore,

$$
x E y \Longleftrightarrow f(x) \Delta f(y) \in I_{\max } \Longleftrightarrow f(x) \Delta f(y) \in I_{\xi} \Longleftrightarrow f(x) E_{I_{\xi}} f(y)
$$

So $E$ Borel reduces to $E_{I_{\xi}}$ by $f$.

Kanovei asked ([8, Question 1] and [9, Question 1]), building on work by Greg Hjorth, if all Borel ideals were Borel stable, which, in particular, would imply that $T_{2}$, a.k.a. $=^{+}$, i.e., equality of countable sets of reals would not Borel reduce to any Borel ideal. Theorem 2 shows this not to be the case.

§3. Operations on quasiorders and equivalence relations. We will in this section define what we call operations on equivalence relations and quasiorders, though we will only be interested in definable relations, e.g., Borel or analytic. The operations we consider will be of the type $R \mapsto R^{\prime}$, associating to each either equivalence relation or quasiorder another equivalence relation or quasiorder. This could be for example the infinite power of the first relation. But a natural constraint on the operation will be that of preserving $\leq_{B}$, i.e., $R \leq_{B} S \Longrightarrow R^{\prime} \leq_{B} S^{\prime}$. This constraint will then guarantee that the operation will be an operation on the degrees, that is, will induce an operation on the $\sim_{B}$ classes. We can therefore speak about jumps, as those operations that are strictly increasing with respect to $\leq_{B}$, i.e., such that $R<{ }_{B} R^{\prime}$

Let us mention first the very important Borel anti-diagonalisation result of Harvey Friedman (see the article by L. Stanley [18]):

Theorem 3. (Friedman) Let E be a Borel equivalence relation on a Polish space $\mathfrak{X}$, and define another equivalence relation $E^{+}$on $\mathfrak{X}^{\omega}$ as follows:

$$
\vec{x} E^{+} \vec{y} \Longleftrightarrow \forall n \exists m, k\left(x_{n} E y_{m} \wedge y_{n} E x_{k}\right)
$$

Then for any Borel function $G: \mathfrak{X}^{\omega} \rightarrow \mathfrak{X}$ such that $\vec{x} E^{+} \vec{y} \Longrightarrow G(\vec{x}) E G(\vec{y})$, there is an $\vec{x}$ and an $n<\omega$ with $G(\vec{x}) E x_{n}$.

The + operation in fact naturally defines a jump operation on Borel equivalence relations and it is shown by Friedman and Stanley in [5, section (1.2)] that if one begins with identity of reals and defines the transfinite iterates of the jump by taking infinite products at limit ordinals, then the corresponding hierarchy will be $\leq_{B}$-cofinal among Borel equivalence relations that Borel reduce to a Borel action of the infinite symmetric group $S_{\infty}$. But unfortunately already $E_{1}$ escapes this picture, as it does not reduce to any relation in this hierarchy, in fact, it does not even Borel reduce to any Polish group action (see Kechris and Louveau [12]). So we are far from having a cofinal family. This we can remedy now that we know that biembeddability of countable combinatorial trees is analytic complete. So our goal here is to extend the analysis done by Friedman and Stanley in [5] and [4] to quasiorders in order to get cofinal families of Borel equivalence relations and quasiorders. 
Definition 4. Let $R$ be a Borel quasiorder on a Polish space $\mathfrak{X}$ and define another quasiorder $R^{c f}$ on $\mathfrak{X}^{\omega}$ as follows: $\vec{x} R^{c f} \vec{y} \Longleftrightarrow \forall n \exists m x_{n} R y_{m}$, i.e., if $\vec{y}$ is $R$-cofinal with $\vec{x}$.

First we deduce the following result essentially contained in [5] (see section $(1.2 .2))$ :

Proposition 5. Suppose $R$ is a Borel quasiorder on a Polish space $\mathfrak{X}$ and $f: \mathfrak{X}^{\omega} \rightarrow$ $\mathfrak{X}$ is a Borel function such that

$$
\begin{aligned}
f(\vec{x}) R f(\vec{y}) & \Longrightarrow \vec{x} R^{c f} \vec{y}, \\
\vec{x}\left(\equiv_{R}\right)^{+} \vec{y} & \Longrightarrow f(\vec{x}) \equiv_{R} f(\vec{y}) .
\end{aligned}
$$

Then for any $\vec{x} \in \mathfrak{X}^{\omega}$ there is an $n<\omega$ with $f(\vec{x}) R x_{n}$.

Proof. Assume towards a contradiction that there is some $\vec{u} \in \mathfrak{X}^{\omega}$ with $\forall i \neg f \vec{u} R u_{i}$. Let

$$
B:=\left\{f \vec{x} \mid \vec{x} \in \mathfrak{X}^{\omega} \wedge \forall i \neg f \vec{x} R x_{i}\right\} \ni f \vec{u}
$$

So

$$
\begin{aligned}
f \vec{x} \equiv_{R} f \vec{z} \in B & \Longrightarrow \forall n \exists m x_{n} R z_{m} \wedge \forall i \neg f \vec{z} R z_{i} \wedge f \vec{x} \equiv_{R} f \vec{z} \\
& \Longrightarrow \forall i \neg f \vec{x} R x_{i} \Longrightarrow f \vec{x} \in B .
\end{aligned}
$$

We now define a Borel function $G:\left(\mathfrak{X}^{\omega}\right)^{\omega} \rightarrow \mathfrak{X}^{\omega}$ by

$$
G\left(\left(\vec{x}_{m}\right)\right)_{n}= \begin{cases}f \vec{u} & \text { if } n=0, \\ f \vec{x}_{n-1} & \text { if } f \vec{x}_{n-1} \in B \text { and } n>0, \\ f \vec{u} & \text { otherwise. }\end{cases}
$$

Notice that for each $X=\left(\vec{x}_{n}\right) \in\left(\mathfrak{X}^{\omega}\right)^{\omega}$, each entry of the sequence $G(X)$ belongs to the set $B$ and, moreover, $f \vec{u}$ is the first term of $G(X)$.

Now, if for some $X=\left(\vec{x}_{n}\right), Z=\left(\vec{z}_{n}\right) \in\left(\mathfrak{X}^{\omega}\right)^{\omega}$, we have $X\left(\equiv_{R}\right)^{++} Z$, then

$$
\forall n \exists m, k \quad \vec{x}_{n}\left(\equiv_{R}\right)^{+} \vec{z}_{m} \wedge \vec{z}_{n}\left(\equiv_{R}\right)^{+} \vec{x}_{k} .
$$

And for such $n, m, k$ we have $f \vec{x}_{n} \equiv_{R} f \vec{z}_{m} \wedge f \vec{z}_{n} \equiv_{R} f \vec{x}_{k}$, whence $f \vec{x}_{n} \in B \Longleftrightarrow$ $f \vec{z}_{m} \in B$ and $f \vec{z}_{n} \in B \Longleftrightarrow f \vec{x}_{k} \in B$.

This implies that if $X\left(\equiv_{R}\right)^{++} Z$ and $f \vec{x}_{n}$ is a term in the sequence $G(X)$, then there is $m$ such that $f \vec{x}_{n} \equiv_{R} f \vec{z}_{m}$, whence also $f \vec{z}_{m}$ is a term in $G(Z)$. Similarly the other way around. In other words, if $X\left(\equiv_{R}\right)^{++} Z$, then also $G(X)\left(\equiv_{R}\right)^{+} G(Z)$. Thus, $G$ and $\left(\equiv_{R}\right)^{+}$verify the hypothesis in Friedman's theorem and there are therefore an $X=\left(\vec{x}_{m}\right) \in\left(\mathfrak{X}^{\omega}\right)^{\omega}$ and an $n$ such that $G(X)\left(\equiv_{R}\right)^{+} \vec{x}_{n}$.

Now either $f \vec{x}_{n} \in B$, whereby $f \vec{x}_{n}$ is a term in $G(X)$ and therefore both $\forall i \neg f \vec{x}_{n} R x_{n}^{i}$ and $\exists i f \vec{x}_{n} R x_{n}^{i}$, which is impossible. Or $f \vec{x}_{n} \notin B$, whence $\exists i f \vec{x}_{n} R x_{n}^{i}$. But, by definition of $n$, there is a $k$ such that $f \vec{x}_{k} \in G(X) \subseteq B$ and $x_{n}^{i} \equiv_{R} f \vec{x}_{k}$, or $x_{n}^{i} \equiv_{R} f \vec{u}$.

In the first case, we have $f \vec{x}_{n} R x_{n}^{i} \equiv_{R} f \vec{x}_{k}$ and $\vec{x}_{n} R^{c f} \vec{x}_{k}$. This means in particular that $\exists j f \vec{x}_{k} \equiv_{R} x_{n}^{i} R x_{k}^{j}$, which contradicts that $f \vec{x}_{k} \in B$. And in the second case, we have again $f \vec{x}_{n} R x_{n}^{i} \equiv_{R} f \vec{u}$, and thus $\vec{x}_{n} R^{c f} \vec{u}$, whereby $\exists j f \vec{u} \equiv_{R} x_{n}^{i} R u_{j}$, contradicting that $\vec{u} \in B$ and thus finishing the proof.

Let us see an application of this result: 
Proposition 6. Let $R$ be a Borel quasiorder on a Polish space $\mathfrak{X}$. Suppose that $R$ admits two incompatible elements a, $b$, i.e., $\forall x \neg(x R a \wedge x R b)$. Then $R<_{B} R^{c f}$.

Proof. Suppose that $f: \mathfrak{X}^{\omega} \rightarrow \mathfrak{X}$ is a Borel reduction of $R^{c f}$ to $R$. Then for any $\vec{x} \in \mathfrak{X}^{\omega}$ there is an $n$ with $f \vec{x} R x_{n}$. So in particular $f\left(a^{\omega}\right) R a, f\left(b^{\omega}\right) R b$ and $f\left(a b^{\omega}\right) R a \vee f\left(a b^{\omega}\right) R b$. But since $a^{\omega} R^{c f} a b^{\omega}$ and $b^{\omega} R^{c f} a b^{\omega}$, we have $f\left(a^{\omega}\right) R f\left(a b^{\omega}\right)$ and $f\left(b^{\omega}\right) R f\left(a b^{\omega}\right)$ whereby either $f\left(a^{\omega}\right) R a, f\left(a^{\omega}\right) R b$ or $f\left(b^{\omega}\right) R a, f\left(b^{\omega}\right) R b$, which is a contradiction.

For the reduction just send $x$ to $x^{\omega}$.

It is necessary to have some additional hypothesis on $R$ to get the above result. For if $R$ is simply the reverse ordering on $\omega$ then it is easy to see that any sequence of naturals is classified with respect to $R^{c f}$ by its maximal element.

Furthermore, if $R$ has two incompatible elements $a$ and $b$ then $a^{\omega}$ and $b^{\omega}$ are $R^{c f}$ incompatible. The same holds for infinite products, if one of the factors admits two incompatible elements. So we get an increasing hierarchy by taking transfinite iterations, where limits are defined by infinite sums or products.

Definition 7. Suppose $R$ is a Borel quasiorder on a Polish space $\mathfrak{X}$. Let for $\vec{x}, \vec{y} \in \mathfrak{X}^{\omega}:$

$$
\begin{aligned}
\vec{x} R^{c f} \vec{y} & \Longleftrightarrow \forall n \exists m, k \quad y_{m} R x_{n} R y_{k}, \\
\vec{x} R^{s u b} \vec{y} & \Longleftrightarrow \forall n \exists m \quad x_{n} \equiv_{R} y_{m} .
\end{aligned}
$$

Notice that $R^{c f}, R^{c f i}$ and $R^{s u b}$ are all operations on quasiorders according to our definition.

Proposition 8. Suppose that $R$ is a Borel quasiorder on a Polish space $\mathfrak{X}$ and $S$ is a Borel quasiorder on $\mathfrak{X}^{\omega}$ with $\left(\equiv_{R}\right)^{+} \subseteq S \subseteq R^{c f i}$. If $\equiv_{R}$ has more than one class, then $S \nless_{B} R$. In particular, this holds for both $S=R^{c f i}$ and $S=R^{s u b}$, as $\equiv_{R^{s u b}}=\left(\equiv_{R}\right)^{+}$.

PRoof. If $f: \mathfrak{X}^{\omega} \rightarrow \mathfrak{X}$ is a Borel reduction of $S$ to $R$, one has

$$
f \vec{x} R f \vec{y} \Longleftrightarrow \vec{x} S \vec{y} \Longrightarrow \vec{x} R^{c f i} \vec{y} \Longrightarrow \vec{x} R^{c f} \vec{y}
$$

and

$$
\vec{x}\left(\equiv_{R}\right)^{+} \vec{y} \Longrightarrow \vec{x} \equiv_{S} \vec{y} \Longrightarrow f \vec{x} \equiv_{R} f \vec{y} .
$$

$f$ thus verifies the conditions of Proposition 5, whereby for any $\vec{x} \in \mathfrak{X}^{\omega}$ there is an $n$ such that $f \vec{x} R x_{n}$.

Therefore, for any $x$, we have $f\left(f\left(x^{\omega}\right)^{\omega}\right) R f\left(x^{\omega}\right)$ and

$$
f\left(f\left(x^{\omega}\right)^{\omega}\right) R f\left(x^{\omega}\right) \Longrightarrow f\left(x^{\omega}\right)^{\omega} S x^{\omega} \Longrightarrow f\left(x^{\omega}\right)^{\omega} R^{c f i} x^{\omega} \Longrightarrow f\left(x^{\omega}\right) \equiv_{R} x,
$$

and hence for any $\vec{y} \in \mathfrak{X}^{\omega}, f \vec{y} \equiv_{R} f\left((f \vec{y})^{\omega}\right)$ and

$$
f \vec{y} \equiv_{R} f\left((f \vec{y})^{\omega}\right) \Longrightarrow \vec{y} S(f \vec{y})^{\omega} \Longrightarrow \vec{y} R^{c f i}(f \vec{y})^{\omega} \Longrightarrow \forall n y_{n} \equiv_{R} f \vec{y} .
$$

Which means that $\equiv_{R}$ can only have one class.

$\S 4$. Scott derivations and cofinal families of Borel quasiorders. We intend to extend the Scott analysis of isomorphism to an analogue analysis of the embeddability relation. To avoid confusion, we note that by embeddability we simply mean isomorphism with a substructure and reserve the notation $\sqsubseteq$ for this. All our structures will be countably infinite and can therefore always be taken to have 
domain $\omega$; we let $s, t, u, v$ be variables for elements of $\omega^{<\omega}$ and $A, B$ variables for relations on $\omega$, i.e., subsets of $\omega \times \omega$.

Definition 9. We define for all $\xi<\omega_{1}$ a relation $\sqsubseteq_{\xi}$ between elements $(A, s)$ and $(B, t)$ as follows:

- $(A, s) \sqsubseteq_{0}(B, t) \Longleftrightarrow|s|=|t| \wedge \forall i, j\left[A\left(s_{i}, s_{j}\right) \leftrightarrow B\left(t_{i}, t_{j}\right) \wedge s_{i}=s_{j} \leftrightarrow t_{i}=t_{j}\right]$,

- $(A, s) \sqsubseteq_{\xi+1}(B, t) \Longleftrightarrow \forall n \exists m\left(A, s^{\wedge} n\right) \sqsubseteq_{\xi}\left(B, t^{\wedge} m\right)$,

- $(A, s) \sqsubseteq_{\lambda}(B, t) \Longleftrightarrow \forall \xi<\lambda(A, s) \sqsubseteq_{\xi}(B, t)$ for $\lambda$ a limit ordinal.

Moreover, let $A \sqsubseteq_{\xi} B \Longleftrightarrow(A, \emptyset) \sqsubseteq_{\xi}(B, \emptyset)$.

The following is the exact analogue of Scott's theorem for isomorphism (for an excellent survey of this result see the article of Barwise [1]) and is proved the same way.

Theorem 10 (Scott). If $A, B \subset \omega \times \omega$, there is an $\alpha<\omega_{1}$ such that

$$
\forall \beta>\alpha \forall s, t \in \omega^{<\omega}(A, s) \sqsubseteq_{\alpha}(B, t) \Longleftrightarrow(A, s) \sqsubseteq_{\beta}(B, t) .
$$

Moreover, $A \sqsubseteq B \Longleftrightarrow \forall \alpha<\omega_{1} A \sqsubseteq_{\alpha} B$.

It is of course meaningful to restrict $\sqsubseteq$ and $\sqsubseteq \xi$ to a certain class of relations on $\omega$, as for example the class of combinatorial trees, which we denote by $\mathfrak{T}$.

We need the following parametrised version of the basic result on Borel derivatives, (confer (34.13) in [11]):

Let $\mathfrak{Y}$ be a standard Borel space and $\mathfrak{X}=\mathscr{P}(C)$, for some denumerable set $C$, and $D: \mathfrak{Y} \times \mathfrak{X} \rightarrow \mathfrak{X}$ a Borel function, such that for any $y \in \mathfrak{Y}, D_{y}$ is a derivation on $\mathfrak{X}$. Then $\Omega_{D}:=\left\{(y, X) \in \mathfrak{Y} \times \mathfrak{X} \mid D_{y}^{\infty}(X)=\emptyset\right\}$ is $\Pi_{1}^{1}$ and $(y, X) \mapsto|X|_{D_{y}}$ is a $\Pi_{1}^{1}$-rank on $\Omega_{D}$.

Theorem 11. Let $R$ be a Borel quasiorder on a Polish space $\mathfrak{Z}$. Then $R \leq_{B} \sqsubseteq_{\alpha}$ on $\mathfrak{T}$ for some $\alpha<\omega_{1}$.

Proof. Let $T:=\left\{(s, t) \in \omega^{<\omega} \times \omega^{<\omega}|| s|=| t \mid\right\}$, which is the full tree on $\omega \times \omega$ and $\mathfrak{X}:=\mathscr{P}(T)$.

$D: \mathfrak{T}^{2} \times \mathfrak{X} \rightarrow \mathfrak{X}$ is the parametrised Borel derivative defined by:

$$
\begin{aligned}
& D(A, B, X)= \\
\{(s, t) \in X \mid & \forall i, j<|s|\left[\left(s_{i}=s_{j} \leftrightarrow t_{i}=t_{j}\right) \wedge\left(A\left(s_{i}, s_{j}\right) \leftrightarrow B\left(t_{i}, t_{j}\right)\right)\right] \\
\wedge & \left.\forall n \exists m\left(s^{\wedge} n, t^{\wedge} m\right) \in X\right\}
\end{aligned}
$$

Now, for any $A, B \in \mathfrak{T}, X, Y \in \mathfrak{X}$, if $X \subseteq Y$, then $D_{A, B}(X) \subseteq D_{A, B}(Y)$ and $D_{A, B}(X) \subseteq X$. So $D$ is clearly a parametrised Borel derivative. Therefore, $\Omega_{D}:=$ $\left\{(A, B, X) \in \mathfrak{T}^{2} \times \mathfrak{X} \mid D_{A, B}^{\infty}(X)=\emptyset\right\}$ is $\Pi_{1}^{1}$ and $(A, B, X) \mapsto|X|_{D_{A, B}}$ is a $\Pi_{1}^{1}$-rank on $\Omega_{D}$. Since the derivative clearly corresponds to the Scott analysis of embeddability, we have $A \sqsubseteq B$ if and only if $D_{A, B}^{\infty}(T) \neq \emptyset$, that is, if and only if $(A, B, T) \notin \Omega_{D}$.

Let now $f: \mathfrak{Z} \rightarrow \mathfrak{T}$ be a Borel reduction of $R$ to $\sqsubseteq$. This can be found as $\sqsubseteq$ on $\mathfrak{T}$ is a complete analytic quasiorder, as shown by Louveau and the author in [16, section (1.2)]. Then, as $R$ is Borel, $(f \times f)[\complement R] \subseteq \complement\{(A, B) \mid A \sqsubseteq B\}$ is analytic, so $(f \times f)[C R] \times\{T\} \subseteq \Omega_{D}$ must be of bounded rank $\alpha$. So by construction of the derivative $\neg x R y \Longleftrightarrow \neg f x \sqsubseteq f y \Longleftrightarrow(f x, f y, T) \in \Omega_{D} \Longleftrightarrow D^{\alpha}(f x, f y, T)=$ $\emptyset \Longleftrightarrow \neg f x \sqsubseteq_{\alpha} f y$. 
Definition 12. Let $R$ be a Borel quasiorder on a Polish space $\mathfrak{X}$. Then for all $\xi<\omega_{1}$ we define a Borel quasiorder $R_{\xi}$ on a Polish space $\mathfrak{Y}_{\xi}$ as follows:

- $R_{0}:=R$ and $\mathfrak{Y}_{0}:=\mathfrak{X}$.

- If $R_{\xi}$ is defined on $\mathfrak{Y}_{\xi}$, let $\mathfrak{Y}_{\xi+1}:=\mathfrak{Y}_{\xi}^{\omega}$ and $R_{\xi+1}:=R_{\xi}^{c f}$.

- If $R_{\xi}$ is defined on $\mathfrak{Y}_{\xi}$ for all $\xi<\lambda$, where $\lambda$ is a limit ordinal, then $\mathfrak{Y}_{\lambda}:=$ $\prod_{\xi<\lambda} \mathfrak{Y}_{\xi}$ and $\vec{x} R_{\lambda} \vec{y} \Longleftrightarrow \forall \xi<\omega_{1} x_{\xi} R_{\xi} y_{\xi}$.

Let now $\mathfrak{X}:=\mathfrak{T} \times \omega^{<\omega}$. Then the following is easily shown.

Lemma 13. Suppose that $\left(\mathfrak{X}, \sqsubseteq_{\xi}\right) \leq_{B}(\mathfrak{Y}, R)$, for some Borel quasiorder $R$ on a Polish space $\mathfrak{Y}$. Then $\left(\mathfrak{X}, \sqsubseteq_{\xi+1}\right) \leq_{B}\left(\mathfrak{Y}^{\omega}, R^{c f}\right)$.

PRoof. Let $f$ be the given reduction. Then $(A, S) \sqsubseteq_{\xi}(B, t) \Longleftrightarrow f(A, s) R f(B, t)$. So

$$
\begin{gathered}
(A, s) \sqsubseteq_{\xi+1}(B, t) \Longleftrightarrow \forall n \exists m\left(A, s^{\wedge} n\right) \sqsubseteq_{\xi}\left(B, t^{\wedge} m\right) \Longleftrightarrow \\
\forall n \exists m f\left(A, s^{\wedge} n\right) R f\left(B, t^{\wedge} m\right) \Longleftrightarrow\left(f\left(A, s^{\wedge} k\right)\right)_{k<\omega} R^{c f}\left(f\left(B, t^{\wedge} k\right)\right)_{k<\omega}
\end{gathered}
$$

And $g:(A, s) \mapsto\left(f\left(A, s^{\wedge} k\right)\right)_{k<\omega}$ is the desired reduction.

Lemma 14. Suppose that for all $\xi<\lambda\left(\lambda<\omega_{1}\right.$ a limit ordinal $)\left(\mathfrak{X}, \sqsubseteq_{\xi}\right) \leq_{B}$ $\left(\mathfrak{Y}_{\xi}, R_{\xi}\right)$, for some Borel quasiorder $R$ on a Polish space $\mathfrak{Y}$. Then $\left(\mathfrak{X}, \sqsubseteq_{\lambda}\right) \leq_{B}$ $\left(\mathfrak{Y}_{\lambda}, R_{\lambda}\right)$.

Proof. Let $f_{\xi}$ be the respective Borel reductions. Then

$$
\begin{gathered}
(A, s) \sqsubseteq_{\lambda}(B, t) \Longleftrightarrow \forall \xi<\lambda(A, s) \sqsubseteq_{\xi}(B, t) \Longleftrightarrow \\
\forall \xi<\lambda f_{\xi}(A, s) R_{\xi} f_{\xi}(B, t) \Longleftrightarrow\left(f_{\xi}(A, s)\right)_{\xi<\lambda} R_{\lambda}\left(f_{\xi}(B, t)\right)_{\xi<\lambda} .
\end{gathered}
$$

And $g:(A, s) \mapsto\left(f_{\xi}(A, s)\right)_{\xi_{\lambda}}$ is the desired reduction.

Notice that $\left(\mathfrak{X}, \sqsubseteq_{0}\right) \leq_{B}(\omega,=)$. For $\left(\mathfrak{X}, \sqsubseteq_{0}\right)$ is easily seen to be a Borel equivalence relation with a countable number of classes. This is because a pair $(A, s)$ is completely characterised with respect to $\sqsubseteq_{0}$ by the quantifier-free diagram of $s$ over $A$.

COROLlary 15. If $R$ is a Borel quasiorder having an infinite antichain then $R_{\xi}$, $\xi<\omega_{1}$ is $\leq_{B}$ cofinal among Borel quasiorders. Therefore also $\equiv_{R_{\xi}}, \xi<\omega_{1}$, is cofinal among Borel equivalence relations.

We also mention (as was noticed by A. Louveau) that the above result can be put on its head. Namely, if instead of varying the quasiorder, we vary the domain, keeping the quasiorder fixed, we also get a cofinal family. More specifically, let $\operatorname{Tr}_{\omega}$ be the set of subtrees of the full tree on $\omega$ and define the following relation on it: $T \mathscr{R} S$ if and only if $\exists f: T \rightarrow S$, which is lexicographically strictly increasing. Of course $\mathscr{R}$ is only analytic, but, if one restricts it to the set of trees of some bounded countable height, it becomes Borel, and varying the height up through the countable ordinals, one gets a cofinal family of Borel quasiorders. This is easily shown by a transfinite induction using the preceding results. On the contrary, we cannot claim that the relation of embedding between trees of bounded height is Borel. This is highly doubtful as the general marriage problem or the problem of finding injective choice functions is analytic, non Borel. This problem is avoided in the definition of $\mathscr{R}$ above, as one demands $f$ to be increasing and not only injective. 
$\S 5$. Complete $K_{\sigma}$ equivalence relations. We begin by elaborating some useful notation introduced by Hjorth and Kechris in [7].

Definition 16. Suppose that $E \subseteq F$ and $R \subseteq S$ are binary relations on Polish spaces $X$ and $Y$ respectively. Write $(E, F) \leq_{B}(R, S)$ iff there is a Borel function $f: X \rightarrow Y$, such that $x E y \Longrightarrow f(x) R f(y)$ and $\neg x F y \Longrightarrow \neg f(x) S f(y)$. This is surely a transitive relation. Furthermore, when writing simply $E$ for the pair $(E, E)$, the relation coincides with the usual Borel reducibility relation.

Let $C=\left\{\left(A_{n}\right) \in \mathscr{P}(\omega)^{\omega} \mid \forall n A_{n} \subseteq A_{n+1}\right\}$ seen as a compact subset of $\left(2^{\omega}\right)^{\omega}$. On $C$ we define the following relations:

$$
\begin{aligned}
\left(A_{n}\right) \leq_{K_{\sigma}}\left(B_{n}\right) & \Longleftrightarrow \exists n \forall m A_{m} \subseteq B_{n+m}, \\
\left(A_{n}\right) H\left(B_{n}\right) & \Longleftrightarrow \exists n \forall m\left[A_{m} \subseteq B_{n+m} \wedge B_{m} \subseteq A_{n+m}\right], \\
\left(A_{n}\right) F\left(B_{n}\right) & \Longleftrightarrow \exists n\left[A_{0} \subseteq B_{n} \vee B_{0} \subseteq A_{n}\right] .
\end{aligned}
$$

Let us recall the following result:

Theorem 17 (Kechris [10], Louveau-Rosendal [16]). The relation $\leq_{K_{\sigma}}$ is a complete $K_{\sigma}$ quasiorder and $H$ is a complete $K_{\sigma}$ equivalence relation.

PRoof. Obviously both $\leq_{K_{\sigma}}$ and $H$ are $K_{\sigma}$ relations.

Suppose $R$ is a $K_{\sigma}$ quasiorder on a Polish space $Y$ and let $\Delta=\{(y, y) \mid y \in Y\}$. Then, as $\Delta$ is closed in $Y^{2}$, the set $\Delta \cap Y^{2}=\Delta$ is a $K_{\sigma}$ set homeomorphic to $Y$, i.e., $Y$ is $K_{\sigma}$. So we can write $Y$ as an increasing union of compact subsets $Y_{n} \subseteq Y$. Write also $R$ as an increasing union of compact relations $F_{n} \subseteq Y^{2}$ and define inductively:

- $R_{0}=\Delta \cap Y_{0}^{2}$,

- $(x, y) \in R_{n+1} \Longleftrightarrow$

$$
x, y \in Y_{n} \wedge\left\{(x, y) \in F_{n} \vee \exists z \in Y_{n}\left((x, z) \in R_{n} \wedge(z, y) \in R_{n}\right)\right\} .
$$

One easily sees that $\left(R_{n}\right)$ is an increasing sequence of compact relations, such that $R_{n} \circ R_{n} \subseteq R_{n+1}$ and $R=\bigcup R_{n}$.

Now fix some basis $\left\{U_{n}\right\}$ for the topology of $Y$ and define for each $n \in \mathbb{N}$ the function $f_{n}: Y \rightarrow \mathscr{P}(\omega)$ by

$$
f_{n}(x)=\left\{k \in \mathbb{N} \mid R_{n}^{x} \cap U_{k} \neq \emptyset\right\} .
$$

As $R_{n}$ is compact, $f_{n}$ is Borel and $f_{n}(x) \subseteq f_{n+1}(x)$.

Now, if $x R y$, then for some $n, x R_{n} y$, and therefore for any $z \in Y$, if $z R_{k} x$, then $z R_{\max (n, k)+1} y$. This shows that $R_{k}^{x} \subseteq R_{n+1+k}^{y}$ and $f_{k}(x) \subseteq f_{n+1+k}(y)$ for all $k$.

Conversely, suppose for some $n$ and all $k, f_{k}(x) \subseteq f_{n+k}(y)$. Take $k$ big enough such that $x R_{k} x$ and notice that $x \in R_{k}^{x} \subseteq R_{n+k}^{y}$, so $x R_{n+k} y$ and $x R y$.

Thus, $\phi: x \mapsto\left(f_{n}(x)\right)$ is a reduction of $R$ to $\leq_{K_{\sigma}}$. Furthermore, if $R$ was actually an equivalence relation, the reduction would also be a reduction of $R$ to $H$.

Suppose in the proof above that $R=H$. Then, as $C$ is compact, we can let $R_{0}=\Delta$ and one sees that $\phi$ reduces $H$ to $(H, F)$.

$H$ is problematic in the sense that it is presented as the equivalence relation associated with a quasiorder and not primarily as the equivalence relation itself. So, in practice, for showing that some equivalence relation is $K_{\sigma}$ complete, it is not of much use, unless the latter is also presented as the equivalence associated with some other complete $K_{\sigma}$ quasiorder. This will be remedied here. We will 
give several, hopefully more handy, versions of this important degree of Borel equivalence relations.

Definition 18. - Let $X_{0}:=\prod_{n \geq 1} n$, where $n=\{0, \ldots, n-1\}$, and define $E_{K_{\sigma}}$ by

$$
\alpha E_{K_{\sigma}} \beta \Longleftrightarrow \exists N \forall k|\alpha(k)-\beta(k)| \leq N .
$$

- See $[\omega]^{\omega}$ as the space of strictly increasing sequences of integers and define $\mathscr{O}$ by

$$
\begin{array}{r}
\left(a_{n}\right) \mathcal{O}\left(b_{n}\right) \Longleftrightarrow \exists N \forall n\left[\#\left(k \mid a_{n}<b_{k}<a_{n+1}\right) \leq N \wedge\right. \\
\left.\#\left(k \mid b_{n}<a_{k}<b_{n+1}\right) \leq N\right] .
\end{array}
$$

- For $\vec{x}, \vec{y} \in \mathbb{R}^{\omega}$ let

$$
\vec{x} E_{\ell_{\infty}} \vec{y} \Longleftrightarrow \vec{x}-\vec{y} \in \ell_{\infty} .
$$

(2) is called the oscillation relation of sequences of integers. It is Stevo Todorcevic's favourite counter example to Ramsey properties for the product, which by itself, of course, is sufficient motivation to classify it.

We say that a relation is essentially of some class $\Gamma$ if it Borel reduces to some relation of class $\Gamma$. It will useful to have some general forms of $K_{\sigma}$ equivalences in mind:

(i) Let $\left(X_{n}, d_{n}\right)$ be a sequence of Polish metric spaces and let for $\alpha, \beta \in \prod X_{n}$ :

$$
\alpha E \beta \Longleftrightarrow \exists N \forall k d_{k}(\alpha(k), \beta(k)) \leq N .
$$

Then $E \leq_{B} H$.

To see this, let $\alpha \mapsto\left(A_{m}^{\alpha}\right)$, where $A_{m}^{\alpha}:=\left\{\beta \in \prod X_{n} \mid \forall k d_{k}(\alpha(k), \beta(k)) \leq m\right\}$ is closed. Then, as $d_{k}$ is a metric, if $\forall k d_{k}(\alpha(k), \beta(k)) \leq N$, we have $A_{k}^{\alpha} \subseteq A_{k+N}^{\beta}$. Conversely, if $A_{0}^{\alpha}=\{\alpha\} \subseteq A_{N}^{\beta}$, then $\forall k d_{k}(\alpha(k), \beta(k)) \leq N$ and $\alpha E \beta$. But now one just replaces each $A_{m}^{\alpha}$ by the set of indices of basic open sets it intersects, which ends the proof.

(ii) Suppose that $\left(f_{n}\right)$ is a sequence of functions from $\omega$ to $\omega$ closed under compositions. Then the following is a quasiorder on $\mathscr{P}(\omega)^{\omega}$ and is essentially $K_{\sigma}$ :

$$
\left(A_{n}\right) \leq_{\vec{f}}\left(B_{n}\right) \Longleftrightarrow \exists N \forall n A_{n} \subseteq B_{f_{N}(n)}
$$

The same holds if we replace $\mathscr{P}(\omega)^{\omega}$ by $\mathscr{F}(X)^{\omega}$ for some Polish space $X$. Furthermore, the corresponding equivalence relation is of course also essentially $K_{\sigma}$.

Proposition 19. $E_{K_{\sigma}}, \mathscr{O}$ and $E_{\ell_{\infty}}$ are Borel bireducible with the complete $K_{\sigma}$ equivalence relation.

PROOF. We will see that they are bireducible with $H$.

Clearly $E_{K_{\sigma}} \leq_{B} E_{\ell_{\infty}}$ and $E_{\ell_{\infty}}$ is essentially $K_{\sigma}$ by (i) above. Also, $\mathscr{O}$ easily extends to a $K_{\sigma}$ equivalence relation on all of $2^{\omega}$.

To show that $H \leq_{B} E_{K_{\sigma}}$, take $g: \omega \times \omega \longleftrightarrow \mathbb{N} \backslash\{0\}$ such that $\left(g^{-1}(p)\right)_{2}<p$, $\forall p \in \mathbb{N} \backslash\{0\}$. Suppose that $A=\left(A_{k}\right)$ is a given increasing sequence of subsets of $\omega$. Then $x_{A}$ is defined as follows: if $g(n, k)=p$ and $q \leq k$ is minimal such that $n \in A_{q}$, or $q=k$ and $n \notin A_{k}$, let $x_{A}(p)=q$.

We now claim that $A \mapsto x_{A}$ is a reduction of $(H, F)$ to $E_{K_{\sigma}}$. 
To see this, suppose that $\neg\left(A_{k}\right) F\left(B_{k}\right)$ and $\forall k \exists n_{k} \in A_{0} \backslash B_{k}$. Then $x_{A}\left(g\left(n_{k}, k\right)\right)=$ 0 and $x_{B}\left(g\left(n_{k}, k\right)\right)=k$ for all $k$, so clearly $x_{B}$ is not bounded by $x_{A}+N$ for any $N$, and therefore $\neg x_{A} E_{K_{\sigma}} x_{B}$.

On the other hand, to show that $\left(A_{k}\right) H\left(B_{k}\right) \Longrightarrow x_{A} E_{K_{\sigma}} x_{B}$, assume that $A_{k} \subseteq$ $B_{k+N}, \forall k$, and let $p=g(n, k)$ be given. We see that if $n \in A_{i}$, then $n \in B_{i+N}$ and $x_{B}(p) \leq i+N$. So if $x_{A}(p) \leq i$, then $x_{B}(p) \leq i+N$. That is, $x_{B} \leq x_{A}+N$. The same proof applies in the other direction.

Finally, we reduce $E_{K_{\sigma}}$ to $\mathscr{O}$ : Let $\left(n_{k}\right)$ be the sequence such that $n_{k+1}-n_{k}=k$, $n_{0}=0$ and $x \mapsto A_{x} \subseteq \omega$ be given by

$$
A_{x} \cap\left[n_{k}, n_{k+1}\left[=\left\{n_{k}, n_{k}+1, \ldots, n_{k}+x(k)\right\} .\right.\right.
$$

Now just enumerate $A_{x}$ in increasing order and one obtains the reduction.

We will now briefly investigate the structure of our newly found candidate for a canonical complete $K_{\sigma}$ equivalence relation. For that we need the notions of isomorphism and invariant embedding.

Let for $E$ and $F$ Borel equivalence relations on Polish spaces $X$ and $Y$ respectively: $E \sqsubseteq_{B} F$ if there is an injective Borel reduction $f$ of $E$ to $F . E \sqsubseteq_{B}^{i} F$ if the $f$ can be chosen injective and such that $f[X]$ is $F$-invariant (i.e., $F$-saturated). And, moreover, $E \cong F$ ( $E$ is isomorphic to $F$ ) if it can be chosen bijective. When we replace the index $B$ by a $c$ it means that the reduction can furthermore be taken continuous. If $G$ is a Borel subset of $X$, we write $E \uparrow_{G}$ for the Borel equivalence relation $E \cap G \times G$. The set $G$ is called a complete section provided it intersects every $E$-class.

Lemma 20. For $E$ a Borel equivalence relation on a Polish space $X$, we have $E_{K_{\sigma}} \leq_{B} E$ iff $E_{K_{\sigma}} \leq_{c} E$.

Proof. This is easiest to see using $\mathscr{O}$. For notice that $E_{K_{\sigma}} \leq_{c} \mathscr{O}$ and that if $f:[\omega]^{\omega} \rightarrow X$ is a Borel reduction of $\mathscr{O}$ to $E$, then $f$ is continuous on some $[A]^{\omega}$ for $A \in[\omega]^{\omega}$. But obviously $\mathscr{O}$ is continuously isomorphic to $\mathscr{O}{ }_{[A]^{\omega}}$.

We notice also that if $E$ is a $K_{\sigma}$ equivalence relation on a compact Polish space $X$, then the reduction of $E$ to $E_{K_{\sigma}}$ given by the proof of Theorem 17 is injective.

A Borel equivalence relation $E$ is called uniformly continuous if $E \cong E \times I_{2^{\omega}}$, where $I_{2^{\omega}}=2^{\omega} \times 2^{\omega}$.

LEMMA 21. $E_{K_{\sigma}}$ is uniformly continuous.

PRoof. Let for $n>1, I_{1}^{n}, \ldots, I_{k_{n}}^{n}$ be a partition of $n$ into intervals of cardinality 2 or 3. For $x, y \in X_{0}$ let $x F y$ iff $\forall n>1 \forall i x(n) \in I_{i}^{n} \leftrightarrow y(n) \in I_{i}^{n}$. Then $F$ is smooth with a continuous selector (taking the lexicographically least element) $s$ and all the classes of $F$ have continuum size. Let $p: X_{0} \times 2^{\omega} \rightarrow X_{0}$ be defined by $p(x, z)=s(x)+z$ (pointwise addition). Then $x F y \rightarrow p(x, z)=p(y, z)$ and $y \neq v \rightarrow p(x, y) \neq p(x, v)$. So by Proposition 3.4 of Kechris and Louveau [12], $E_{K_{\sigma}}$ is uniformly continuous.

Proposition 22. Let $E$ be a uniformly continuous $K_{\sigma}$ equivalence relation on a compact metric space $X$, bireducible with $E_{K_{\sigma}}$. Then $E \cong E_{K_{\sigma}}$.

Proof. We notice that since $E \sqsubseteq_{B} E_{K_{\sigma}}$ and $E$ is uniformly continuous, $E \sqsubseteq_{B}^{i}$ $E_{K_{\sigma}}$ by Lemma 3.2 in Kechris and Louveau [12]. Also as $E_{K_{\sigma}} \leq_{B} E$ we have $E_{K_{\sigma}} \sqsubseteq_{B} E \times I_{2^{\omega}}$ and therefore again $E_{K_{\sigma}} \sqsubseteq_{B}^{i} E \times I_{2^{\omega}} \cong E$. The result now follows by a Schröder-Bernstein argument. 
Though heuristically there can be a big difference between the presentation of an equivalence relation as, for example, an isomorphism relation and the equivalence relation associated to a quasiorder, we will now see that $K_{\sigma}$ quasiorders are in fact somewhat inevitable when dealing with equivalence relations.

Proposition 23. A Borel equivalence relation $E$ on a Polish space $X$ is $K_{\sigma}$ complete iff $E=\equiv_{S}$ for some complete $K_{\sigma}$ quasiorder $S$ on $X$.

Proof. Notice first that as the diagonal $\Delta(X)$ is a closed subset of $E$ it is $K_{\sigma}$ and the space $X$ is therefore also $K_{\sigma}$. Let $f: X_{0} \rightarrow X$ be a continuous reduction of $E_{K_{\sigma}}$ to $E$ and let $R$ be defined on $X_{0}$ by $x R y$ iff there is an $N$ such that for all $n x(n) \leq y(n)+N$. One sees from the proof of Proposition 19 that $R$ reduces $\leq_{K_{\sigma}}$ and is therefore $K_{\sigma}$ complete. Put $P=(f \times f)[R]$ which is then $K_{\sigma}$ by the continuity of $f$. Let for $x, y \in X$ :

$$
x S y \Leftrightarrow \exists z, v \in X(z E x \wedge v E y \wedge z P v) \vee x E y .
$$

As $X$ and $P$ are $K_{\sigma}$, so is $S$. Moreover, one can check that $S$ is a quasiorder, reduces $R$ and $\equiv_{S}=E$.

This is the analogue of a similar result for complete $\Sigma_{1}^{1}$ equivalence relations from [16].

Two metric spaces $\left(X, d_{X}\right)$ and $\left(Y, d_{Y}\right)$ are said to be Lipschitz isomorphic (or Lipschitz homeomorphic, in symbols $\left.\left(X, d_{X}\right) \sim_{L}\left(Y, d_{Y}\right)\right)$ if there are a homeomorphism $f: X \longleftrightarrow Y$ and a constant $c \geq 1$, such that for all $x, y \in X \frac{1}{c} d_{X}(x, y) \leq$ $d_{Y}(f x, f y) \leq c d_{X}(x, y)$. Write $\left(X, d_{X}\right) \sim_{L}^{c}\left(Y, d_{Y}\right)$ if it holds for the constant $c$.

Note that such a function will preserve Cauchy sequences in both directions, and therefore to check whether two Polish metric spaces are Lipschitz isomorphic it is enough to verify that they have Lipschitz isomorphic countable dense subsets.

This implies that if one sees Polish metric spaces as the set of closed subspaces of some universal Polish metric space, as, for example, the Urysohn space $\mathbb{U}$ (confer the articles by Clemens, Gao and Kechris [3, 6]), then the notion of Lipschitz isomorphism becomes analytic in the Effros Borel structure.

THEOREM 24. Lipschitz isomorphism of compact metric spaces is Borel bireducible with $E_{K_{\sigma}}$.

Proof. We commence by reducing Lipschitz isomorphism to some $K_{\sigma}$ equivalence of the form (ii) following Definition 18. This will follow along the lines of Gromov's proof that isometry of compact metric spaces is smooth.

Notice that if $f:\left(K, d_{K}\right) \longleftrightarrow\left(L, d_{L}\right)$ is a $c$-Lipschitz isomorphism, then any $t$-net in $K$ (i.e., a set such that any point in $K$ is of distance less than $t$ to some point in the set) will be sent to a $c t$-net in $L$.

If $\vec{x} \in K^{n}$, we let $b(\vec{x})$ be the distance matrix $\left[d_{K}\left(x_{i}, x_{j}\right)\right] \in \mathbb{R}^{n \times n}$. Moreover, we let $D_{n, t}(K) \subset \mathbb{R}^{n \times n}$ be the set of distance matrices of $n$-tuples that are $t$-nets in $K$.

Suppose that $A=\left[a_{i j}\right] \in \mathbb{R}^{n \times n}$. Then a $d$-perturbation, $d \geq 1$, of $A$ is a matrix $\left[d_{i j} a_{i j}\right]$, where $\frac{1}{d} \leq d_{i j} \leq d$.

Let $E_{n, t, d}(K)$ be the set of $d$-perturbations of elements in $D_{n, t}(K)$. This is a compact subset of $\mathbb{R}^{n \times n}$. Let also $\mathbb{D}=\mathbb{N}^{*} \times \mathbb{Q}^{+} \times \mathbb{Q} \cap[1,+\infty[$ and let the function

$$
\Theta: \mathscr{K}(\mathbb{U}) \longrightarrow \prod_{(n, t, d) \in \mathbb{D}} \mathbb{R}^{n \times n}
$$

be defined by $\Theta(K)=\left(E_{n, t, d}(K)\right)_{(n, t, d) \in \mathbb{D}}$. It is easily checked that $\Theta$ is Borel. 
We claim that $\left(K, d_{k}\right) \sim_{L}\left(L, d_{L}\right)$ if and only if

$$
\exists c \in \mathbb{Q} \cap\left[1,+\infty\left[\forall(n, t, d) \in \mathbb{D} E_{n, t, d}(K) \subseteq E_{n, c t, c d}(L) .\right.\right.
$$

For $c \in \mathbb{Q} \cap\left[1,+\infty\left[\right.\right.$ let $g_{c}: \mathbb{D} \rightarrow \mathbb{D}$ be defined by $g_{c}(n, t, d)=(n, c t, c d)$. The functions $g_{c}, c \in \mathbb{Q} \cap[1,+\infty$ [ are clearly closed under compositions, so given our claim this shows $\sim_{L}$ to reduce to something of the form (ii).

So suppose $\left(K, d_{K}\right) \sim_{L}\left(L, d_{L}\right)$, by some $f$ of Lipschitz constant $c$. This implies $E_{n, t, d}(K) \subseteq E_{g_{c}(n, t, d)}(L)$ and $E_{n, t, d}(L) \subseteq E_{g_{c}(n, t, d)}(K)$ and shows one direction.

Suppose on the other hand that $E_{n, t, d}(K) \subseteq E_{g_{c}(n, t, d)}(L)$ for all $n, t, d$. Then clearly (taking $d=1$ ) for any $t$-net $\vec{x} \in K^{n}$ there is a $c t$-net $\vec{y} \in L^{n}$ with $\vec{x} \sim_{L}^{c} \vec{y}$ (i.e., sending $x_{i}$ to $y_{i}$ ).

Let $\left(x_{i}\right)$ be a countable dense set in $K$ and take for all $n$ a $\vec{y}_{n}=\left\{y_{0}^{n}, \ldots, y_{n}^{n}\right\}$ $\in L^{n+1}$ such that

$$
\left\{x_{0}, \ldots, x_{n}\right\} \sim_{L}^{c}\left\{y_{0}^{n}, \ldots, y_{n}^{n}\right\}
$$

and

$$
d_{H}\left(\left\{y_{0}^{n}, \ldots, y_{n}^{n}\right\}, L\right)<2 c d_{H}\left(\left\{x_{0}, \ldots, x_{n}\right\}, K\right) .
$$

Here $d_{H}\left(\left\{x_{0}, \ldots, x_{n}\right\}, K\right)$ is simply the Hausdorff distance between $\left\{x_{0}, \ldots, x_{n}\right\}$ and $K$, which in this case is the supremum of the distance of a point in $K$ to $\left\{x_{0}, \ldots, x_{n}\right\}$, that is, in some sense $\left\{x_{0}, \ldots, x_{n}\right\}$ 's modulus of density.

Diagonalising and using the fact that $L$ is compact, one can find an infinite $A \subset \omega$ and $y_{i} \in L$ such that $y_{i}^{n} \underset{n \in A}{\longrightarrow} y_{i}$ for all $i$. Then as $\left\{x_{0}, \ldots, x_{n}\right\} \sim_{L}^{c}\left\{y_{0}^{n}, \ldots, y_{n}^{n}\right\}$ we have that $\left(x_{i}\right) \sim_{L}^{c}\left(y_{i}\right)$, so $K$ is $c$-Lipschitz isomorphic with a closed subset of $L$.

We shall see that it is surjective. Otherwise, as the image of $K$ is closed in $L$, there is an open ball $B(y, r) \subseteq L,(r>0)$, not intersecting the image of $K$. Take $n$ such that $\left\{x_{0}, \ldots, x_{n}\right\}$ is a $\frac{r}{6 c}$-net and $m \geq n, m \in A$, such that

$$
\forall i \leq n \quad d_{L}\left(y_{i}^{m}, y_{i}\right)<\frac{r}{3} \text {. }
$$

Using now that for all $k$

$$
d_{H}\left(\left\{y_{0}^{k}, \ldots, y_{k}^{k}\right\}, L\right)<2 c d_{H}\left(\left\{x_{0}, \ldots, x_{k}\right\}, K\right)
$$

and, a fortiori, $\left\{x_{0}, \ldots, x_{m}\right\}$ is a $\frac{r}{6 c}$-net, one has that $\left\{y_{0}^{m}, \ldots, y_{m}^{m}\right\}$ is a $\frac{r}{3}$-net. So there is a $j \leq m$ with $d_{L}\left(y_{j}^{m}, y\right)<\frac{r}{3}$.

Again by density there is an $i \leq n$ with $d_{K}\left(x_{i}, x_{j}\right)<\frac{r}{3 c}$, whereby as

$$
\left\{x_{0}, \ldots, x_{m}\right\} \sim_{L}^{c}\left\{y_{0}^{m}, \ldots, y_{m}^{m}\right\}
$$

one has $d_{L}\left(y_{i}^{m}, y_{j}^{m}\right)<\frac{r}{3}$. So

$$
d_{L}\left(y, y_{i}\right) \leq d_{L}\left(y, y_{j}^{m}\right)+d_{L}\left(y_{j}^{m}, y_{i}^{m}\right)+d_{L}\left(y_{i}^{m}, y_{i}\right)<\frac{r}{3}+\frac{r}{3}+\frac{r}{3} \leq r .
$$

But this is impossible, as $y_{i}$ is in the image of $K$, and the image of $K$ has therefore to be $L$. This finishes the proof of the claim.

We are now left with showing that Lipschitz isomorphism is complete. This will be done by rendering $E_{K_{\sigma}}$ more rigid.

We work in $\mathbb{R}^{2}$ and define $A=\{0\} \times[0,1], B=[0,1] \times\{0\}$ and for $n \in \mathbb{N}, k \in \mathbb{N}^{*}$ let $C_{k}^{n}=\left\{\frac{1}{k}\right\} \times\left[0, \frac{1}{e^{n}}\right]$. 
Let now $f: X_{0} \rightarrow \mathscr{K}\left(\mathbb{R}^{2}\right)$ be defined by: $f \vec{x}=A \cup B \cup \bigcup_{n \in \mathbb{N}^{*}} C_{n}^{\vec{r}(n)}$.

If now $f \vec{x} \sim_{L}^{c} f \vec{y}$, then, a fortiori, they are homeomorphic and it is therefore easy to see, by checking the branching points, that $C_{n}^{\vec{x}(n)}$ must be sent bijectively to $C_{n}^{\vec{y}(n)}$. So, in particular,

$$
\frac{e^{-x(k)}}{e^{-y(k)}}=e^{y(k)-x(k)} \leq c, \quad \frac{e^{-y(k)}}{e^{-x(k)}}=e^{x(k)-y(k)} \leq c .
$$

Therefore, $|y(k)-x(k)| \leq \log c$ and $\vec{x} E_{K_{\sigma}} \vec{y}$.

For the other direction just send $A$ to $A, B$ to $B$ and $C_{n}^{\vec{r}(n)}$ to $C_{n}^{\vec{\gamma}(n)}$. Then the above calculation shows it to be a Lipschitz isomorphism in case $\vec{x} E_{K_{\sigma}} \vec{y}$.

We mention that one can in fact show that $E_{1}$ Borel reduces to Lipschitz isomorphism of separable Banach spaces [17]. But in fact for the case of general Polish metric spaces it is not difficult to show that Lipschitz isomorphism also reduces the universal $S_{\infty}$ action.

Proposition 25. $E_{S_{\infty}}^{\infty} \leq_{B}$ 'Lipschitz isomorphism of Polish metric spaces'.

Proof. We reduce isomorphism of countable combinatorial trees to $\sim_{L}$, knowing that this relation is Borel bireducible with $E_{S_{\infty}}^{\infty}$ (see Friedman and Stanley [5, theorem (1.1.1)]) . Here a countable combinatorial tree is simply a connected, symmetric, irreflexive binary relation on $\omega$ without cycles.

First for any combinatorial tree $T \subset \omega \times \omega$ and any node in $T$ we add two infinite branches parting from the node. Call the tree so obtained $\tilde{T}$.

Obviously $T \simeq S \Longrightarrow \tilde{T} \simeq \tilde{S}$, but also $\tilde{T} \simeq \tilde{S} \Longrightarrow T \simeq S$ and any isomorphism between $\tilde{T}$ and $\tilde{S}$ restricts to an isomorphism of $T$ and $S$. This is so as any point $x \in T \subset \tilde{T}$ has valency $\geq 3$ in $\tilde{T}$ and any point $x \in \tilde{T} \backslash T$ has valency 2 . Correspondingly for $S$ and $\tilde{\tilde{S}}$.

Now replace any edge in $\tilde{T}$ by the line segment $[0,1]$ and equip the object, $T^{\prime}$, so obtained with the geodesic distance. So $T^{\prime}$ is the $\mathbb{R}$-tree spanned by $\tilde{T}$. Then we have the following:

$$
\left.\forall x \in \tilde{T} \subseteq T^{\prime} \quad \forall \varepsilon, \delta \in\right] 0,1[[\operatorname{valency}(x)=\# \partial B(x, \varepsilon)=\# \partial B(x, \delta)] .
$$

The valency of a point $x \in \tilde{T} \subseteq T^{\prime}$ is therefore the smallest natural number such that $x$ has a neighborhood basis consisting of sets whose boundary is exactly of that cardinality.

This means that the valency is a topological invariant for the points in $\tilde{T}$ and is therefore preserved under Lipschitz isomorphism. Moreover, any Lipschitz isomorphism between $T^{\prime}$ and $S^{\prime}$ will of course send the branching points to the branching points and therefore finally send $T$ to $S$. But the points in $T^{\prime}$ and $S^{\prime}$ are uniquely path connected, so this path will also preserved. So all in all this implies that the Lipschitz isomorphism restricts to an isomorphism of $T$ and $S$.

This of course gives an indication of Lipschitz isomorphism being monstrously complicated and it seems to be the best candidate for an isomorphism relation being analytic complete. For we know both that it reduces relatively complicated Polish group actions and that it cannot itself reduce to a Polish group action as it reduces $E_{K_{\sigma}}$ and therefore $E_{1}$. But our methods for showing something to be analytic complete are yet not sufficiently developed for direct attack on this problem. 
We finish by classifying the relation of equivalence of Schauder bases. Our space of bases will be chosen as Bossard does in [2], where a basis is seen as a subsequence of the universal basis, $\left\{u_{n}\right\}_{\omega}$, constructed by Pełczynski. That is, any Schauder basis is equivalent to a subsequence of Pekczynski's basis and can therefore be identified with some $\left\{u_{n}\right\}_{A}, A \in[\omega]^{\omega}$. We note that Bossard in the above mentioned paper shows that $E_{0}$ Borel reduces to equivalence of bases.

Proposition 26. Equivalence of Schauder bases is Borel bireducible with $E_{K_{\sigma}}$.

The following lemma is classical and was shown to me by Olivier Guédon.

Lemma 27. For $1<p<q<\infty$ and $n \in \mathbb{N}$, we have

$$
\sup \left(\frac{\left\|\left(x_{1}, \ldots, x_{n}\right)\right\|_{p}}{\left\|\left(x_{1}, \ldots, x_{n}\right)\right\|_{q}} \mid x_{1}, \ldots, x_{n} \in \mathbb{R}\right)=\frac{\left(1^{p}+\cdots+1^{p}\right)^{1 / p}}{\left(1^{q}+\cdots+1^{q}\right)^{1 / q}}=n^{\frac{1}{p}-\frac{1}{q}} .
$$

Proof. Let $t p=q$ and $t^{\prime}$ be its conjugate exponent, i.e., $\frac{1}{t}+\frac{1}{t^{\prime}}=1$. So $\frac{1}{p t^{\prime}}=\frac{1}{p}-\frac{1}{q}$ and $t^{\prime}=\frac{q}{q-p}$ and by Holder's inequality one has for positive $a_{1}, \ldots, a_{n}$

$$
\begin{gathered}
\left(\sum a_{i}^{p}\right)^{1 / p}=\left(\sum a_{i}^{p} \cdot 1\right)^{1 / p} \leq\left[\left(\sum a_{i}^{p t}\right)^{1 / t}\left(\sum 1^{t^{\prime}}\right)^{1 / t^{\prime}}\right]^{1 / p}= \\
\left(\sum a_{i}^{q}\right)^{1 / q} \cdot n^{1 / p t^{\prime}}=\left(\sum a_{i}^{q}\right)^{1 / q} \cdot n^{1 / p-1 / q} .
\end{gathered}
$$

So the supremum of the difference of the norms is attained on the diagonal $\left(a_{1}, \ldots, a_{n}\right)=(1, \ldots, 1)$.

And now to the proof of the proposition.

Proof. First we show that equivalence of bases, $\sim$, Borel reduces to $E_{K_{\sigma}}$.

For $\vec{s} \in(\mathbb{Q} \cap[-1,1])^{<\omega}, c \geq 1$ and $A=\left\{a_{0}, a_{1}, a_{2}, \cdots\right\}<\in[\omega]^{\omega}$ put

$$
\begin{aligned}
d(A, \vec{s}) & =\left\|s_{0} u_{a_{0}}+\cdots+s_{|s|-1} u_{a_{|s|-1}}\right\|, \\
D(A, \vec{s}, c) & =\left[\frac{1}{c} d(A, \vec{s}), c d(A, \vec{s})\right] .
\end{aligned}
$$

So if $A \sim B$, there is an $r \in \mathbb{Q} \cap\left[1,+\infty\left[\right.\right.$ such that for all $\vec{s} \in(\mathbb{Q} \cap[-1,1])^{<\omega}$ and $c \geq 1$

$$
\begin{aligned}
& D(A, \vec{s}, c) \subseteq D(B, \vec{s}, r c), \\
& D(B, \vec{s}, c) \subseteq D(A, \vec{s}, r c) .
\end{aligned}
$$

On the other hand, if this is the case, then, in particular,

$$
d(A, \vec{s}) \in D(A, \vec{s}, 1) \subseteq D(B, \vec{s}, r) .
$$

Therefore, $\forall \vec{s} \in(\mathbb{Q} \cap[-1,1])^{<\omega}$, we have

$$
\begin{aligned}
\frac{1}{r}\left\|s_{0} u_{b_{0}}+\cdots+s_{|s|-1} u_{b_{|s|-1}}\right\| & \leq\left\|s_{0} u_{a_{0}}+\cdots+s_{|s|-1} u_{a_{|s|-1}}\right\| \\
& \leq r\left\|s_{0} u_{b_{0}}+\cdots+s_{|s|-1} u_{b_{|s|-1}}\right\|
\end{aligned}
$$

and the two sequences are equivalent. This shows that $\sim$ is of the form $(\star)$.

For the other direction one notices that Lemma 27 permits us to choose for each $k$ an $n$ and $1<p_{0}<\cdots<p_{k-1}<\infty$, such that the constant of equivalence between $\ell_{p_{i}}^{n}$ and $\ell_{p_{j}}^{n}, i<j$, is exactly $2^{j-i}$. Do this and call them $n(k)$ and $1 \leq p(k, 0)<\cdots<p(k, k-1) \leq \infty$ respectively. The mapping 


$$
\vec{x} \mapsto\left(\ell_{p\left(0, x_{0}\right)}^{n(0)} \oplus \ell_{p\left(1, x_{1}\right)}^{n(1)} \oplus \ell_{p\left(2, x_{2}\right)}^{n(2)} \oplus \cdots\right)_{\ell_{1}}
$$

is then a reduction from $E_{K_{\sigma}}$ on $X_{0}$ to the relation of equivalence of Schauder bases.

Let us end with a list of problems left open:

Problem 28. - Determine the complexity of Lipschitz isomorphism of Polish metric spaces. In particular, is it $\boldsymbol{\Sigma}_{1}^{1}$-complete?

- Are all $F_{\sigma}$ equivalence relations Borel reducible to $E_{K_{\sigma}}$ ?

- More modestly, are all Borel equivalence relations with $K_{\sigma}$ classes Borel reducible to $E_{K_{\sigma}}$ ?

- (Louveau) What about $F_{\sigma}$ equivalence relations that can be written as $\bigcup F_{n}$, where the $F_{n}$ are closed symmetric relations with $F_{0}=\Delta$ and $F_{n} \circ F_{n} \subseteq F_{n+1}$ ? Notice that in this case there is a natural C-measurable reduction to $E_{K_{\sigma}}$.

\section{REFERENCES}

[1] J. BARWISE, Back and forth through infinitary logic, Studies in model theory, Mathematical Association of America, 1973.

[2] B. Bossard, A coding of separable Banach spaces. Analytic and coanalytic families of Banach spaces, Fundamenta Mathematicae, vol. 172 (2002), pp. 117-152.

[3] J. Clemens, S. GaO, and A. Kechris, Polish metric spaces: their classification and isometry groups, The Bulletin of Symbolic Logic, vol. 7 (2001), pp. 361-375.

[4] H. Friedman, Borel and Baire reducibility, Fundamenta Mathematicae, vol. 164 (2000), pp. 61-69.

[5] H. Friedman and L. STANLey, A Borel reducibility theory for classes of countable structures, this JOURNAL, vol. 54 (1989), pp. 894-914.

[6] S. GAO and A. KECHRIS, On the classification of Polish metric spaces up to isometry, vol. 161, Memoirs of the American Mathematical Society, no. 766, 2003.

[7] G. HJorth and A. KeCHRIs, Analytic equivalence relations and Ulm-type classifications, this JOURNAL, vol. 60 (1995), pp. 1273-1300.

[8] V. KANOVEI, Varia of ideals and ERs, forthcoming book.

[9] V. KANOVEI and M. REEKEN, Some new results on Borel irreducibility of equivalence relations, Izvestiya: Mathematics, vol. 67 (2003), no. 1.

[10] A. KeCHRIS, Lectures on definable group actions and equivalence relations, circulated notes (1994).

[11] _ Classical descriptive set theory, Springer, 1995.

[12] A. Kechris and A. Louveau, The classification of hypersmooth Borel equivalence relations, Journal of the American Mathematical Society, vol. 10 (1997), no. 1, pp. 215-242.

[13] A. Louveau, Analytic partial orders, preprint.

[14] - On the Borel reducibility ordering, preprint.

[15] A. Louveau and C. Rosendal, Relations d'equivalence analytiques completes, Comptes Rendus de l'Académie des Sciences. Série I, vol. 333 (2001), pp. 903-906.

[16] — Complete analytic equivalence relations, vol. 357 (2005), pp. 4839-4866.

[17] C. Rosendal, Etude descriptive de l'isomorphisme dans la classe des espaces de Banach, Ph.D. thesis, Universite Paris 6, 2003.

[18] L. Stanley, Borel diagonalization and abstract set theory; recent results of Harvey Friedman, Harvey Friedman's research on the foundations of mathematics (L.A. Harrington et al., editors), North Holland, 1985, pp. 11-86.

EQUIPE D'ANALYSE FONCTIONNELLE. UNIVERSITÉ PARIS 6. BOÎTE 186, 4 PLACE JUSSIEU 75252 PARIS CEDEX 05, FRANCE

Current address: Department of Mathematics, University of Illinois at Urbana, 273 Altgeld Hall, MC-382, 1409 West Greet Street, Urbana, IL 61801, USA

E-mail: rosendal@ccr.jussieu.fr 